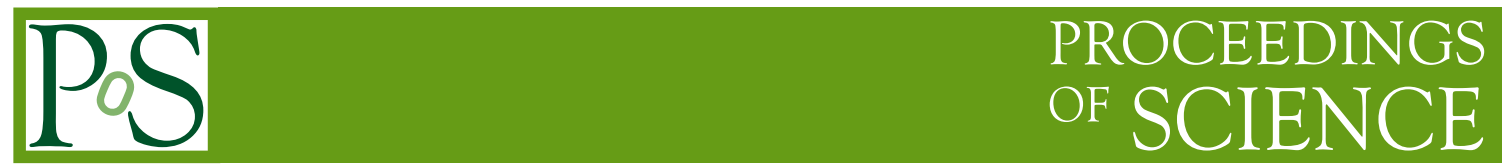

\title{
Jet Reconstruction with charged tracks only in CMS
}

\author{
Paolo Azzurri * \\ Scuola Normale Superiore, piazza dei Cavalieri 7, 56100 Pisa, Italy \\ E-mail: paolo.azzurriecern.ch
}

The performance of jet finding using only charged tracks in CMS has been investigated. Different jet algorithms have been applied to QCD di-jet events, to hadronic $t \bar{t}$ multi-jet events and on $\mathrm{Z}+$ jets events. Results using jets made with tracks only or calorimeter towers are compared for energy response, angular resolution and jet matching to the leading partons. The jet reconstruction performance in the presence of pile-up interactions is presented for the $\mathrm{Z}+\mathrm{jets}$ sample.

2008 Physics at LHC

September 29 - 4 October 2008

Split, Croatia

* on behalf of the CMS Collaboration 


\section{Introduction}

The performance of jet finding in CMS based on clustering the energy deposits of calorimeter towers ("CaloTowers") into calorimeter jets ("CaloJets") using different jet algorithms and jet size parameters is well documented [1,2]. The studies are based on comparisons to jets clustered with the same algorithms with stable Monte Carlo (MC) truth particles as inputs, which are referred to as generator jets ("GenJets").

Besides the fluctuations in the charged component, charged particles represent the jet component that is measured best both in terms of energy resolution and of angular direction, that is extremely well determined at the interaction point. For these reasons, one can expect the tracks picture of a multi-jet event to be more collimated than the CaloTower picture, with less overlap and interference between jets, and less background (e.g. from pile-up events).

With respect to CaloJets, jet reconstruction with charged tracks only ("TrackJets") is a completely independent way to find and count jets, and determine their directions, with independent detector-related systematic uncertainties [3].

\section{Performances in QCD di-jet events}

In order to study the performance of jet reconstruction with charged tracks only, jets are clustered using $a$ ) the standard Iterative Cone (IC) algorithm and $b$ ) the $k_{\mathrm{T}}$ algorithm. The "E-scheme" is used for the clustering recombination. The Iterative Cone algorithm is used with different radii from $R=0.3$, to $R=0.7$. The $k_{\mathrm{T}}$ jets are clustered with size-parameters from $D=0.2$ to $D=0.6$. Reconstructed TrackJets are compared to CaloJets and GenJets using the same schemes.

Results are obtained studying QCD di-jet events where the leading $p_{\mathrm{T}}$ parton is well within the tracker acceptance $(|\eta|<2)$ and is associated to a GenJet. Figure 1 shows the efficiencies to find a reconstructed jet within $\Delta R<0.3$ to the GenJet associated to the leading parton, for CaloJets and TrackJets, using a $k_{\mathrm{T}}$ clustering algorithm with $D=0.6$. TrackJets have a slightly better efficiency than CaloJets at low $p_{\mathrm{T}}$.
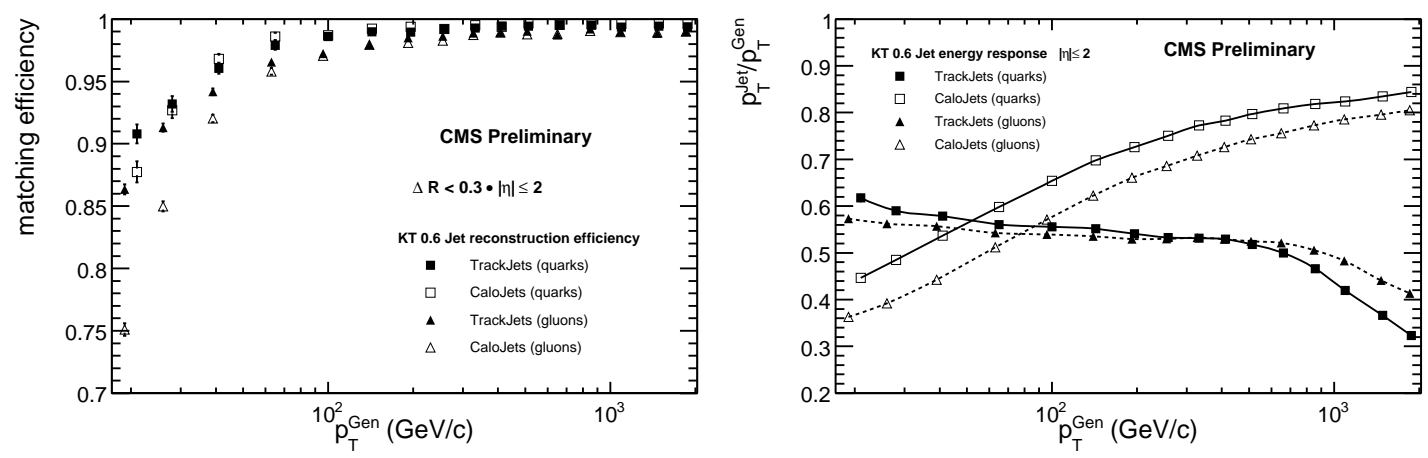

Figure 1: Reconstruction efficiency and $p_{\mathrm{T}}$ response with respect to Monte Carlo truth jets associated to the leading parton in QCD di-jet events, shown separately for jets originating from quarks and gluons.

Figure 1 also shows that the CaloJet $p_{\mathrm{T}}$ response increases almost linearly as a function of $\log p_{\mathrm{T}}$ while the TrackJet response is rather stable around 55\%, decreasing slowly in the $p_{\mathrm{T}}=100$ $\mathrm{GeV} / \mathrm{c}$ to $1 \mathrm{TeV} / \mathrm{c}$ range and dropping for $p_{\mathrm{T}}>1 \mathrm{TeV} / \mathrm{c}$, due to tracking inefficiencies in the core of 
high $p_{\mathrm{T}}$ jets. It also can be seen that the response to gluon jets is lower than for quark jets, because of their wider shower and softer fragmentation function.

The angular resolution of the reconstructed jet axis is evaluated with respect to the direction of the Monte Carlo truth GenJet. TrackJets yield better $\phi$ resolutions for $p_{\mathrm{T}}<200 \mathrm{GeV} / \mathrm{c}$, while the CaloJets $\phi$ resolutions improve at higher $p_{\mathrm{T}}$. The $\eta$ resolutions of TrackJets and CaloJets are very similar, where the $\eta$ direction of the CaloJets is corrected to the position of the primary interaction vertex determined using the reconstructed tracks.
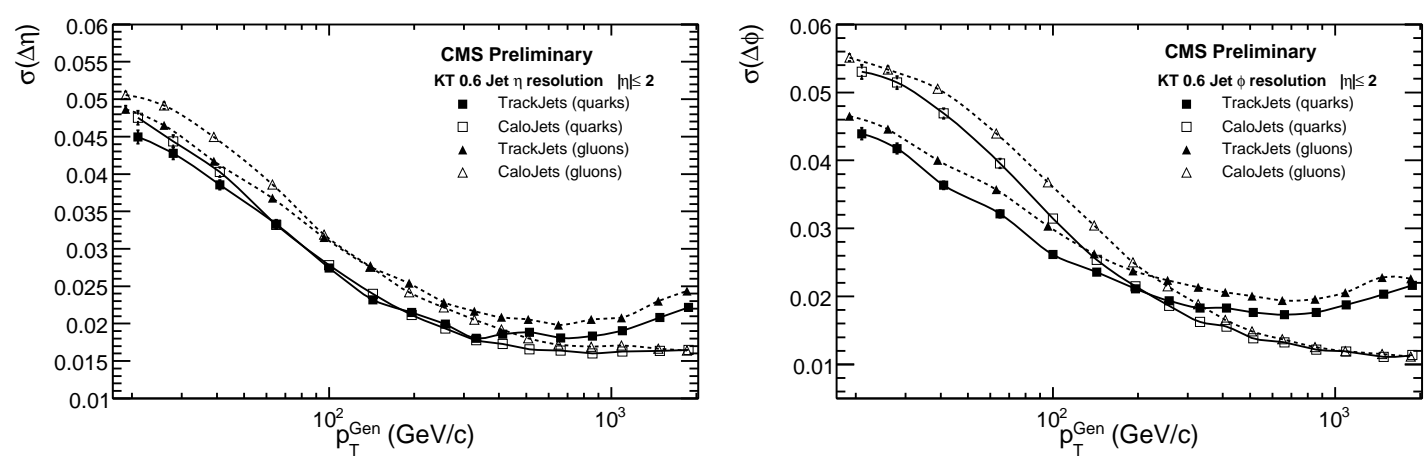

Figure 2: Jet $\eta$ and $\phi$ resolution as a function of the GenJet $p_{\mathrm{T}}$, for calorimeter and TrackJets originating from quarks and gluons. All jets are clustered with the $k_{\mathrm{T}}$ algorithm with $D=0.6$.

\section{Performances in hadronic top pairs decays}

To evaluate the performance of jet clustering with tracks only in a multi-jet environment, samples of fully hadronic decays of $t \bar{t}$ events are analyzed. The final state of these events consists of six quarks $\left(b \bar{b} q \bar{q}^{\prime} q \bar{q}^{\prime}\right)$ that should be reconstructed as six jets.

The matching of jets to quarks is done for each reconstructed jet collection associating each quark to the closest jet in $\Delta R$ and requiring $\Delta R<0.2$. Quarks that match to the same jet indicate that their jets have been merged, so they are removed from the list of matched quarks.

The optimal jet clustering size turns out to be in the $R=0.3-0.5$ range for the cone jets radius and in the $D=0.4-0.6$ range for the $k_{\mathrm{T}}$ jets clustering, and the best matching performances are obtained with TrackJets.

\section{Performances in $Z+$ jets events}

Direct productions of vector bosons have large cross sections at the LHC, and multi-jet events associated with W/Z bosons are relevant to Standard Model (SM) measurements and for searches beyond the SM. In these events TrackJets can provide a robust method for jet counting.

Events where the $\mathrm{Z}$ boson decays into a pair of muons with both muons reconstructed with $p_{\mathrm{T}}(\mu)>20 \mathrm{GeV} / \mathrm{c}$ have been studied. The jet finding efficiency turns out to be significantly better for TrackJets for $p_{\mathrm{T}}<30 \mathrm{GeV} / \mathrm{c}$ and comparable to CaloJets for higher GenJet $p_{\mathrm{T}}$.

Another useful measure of the quality of jet reconstruction is the jet mismatched rate, here defined as the fraction of the reconstructed jets, which are not matched to a generator level jet within $\Delta R<0.3$. 
Figure 3 shows the mismatched jet rate for the TrackJets and CaloJets. The TrackJets give smaller mismatched jet rate (less than a few $\%$ for $p_{\mathrm{T}}>15 \mathrm{GeV} / \mathrm{c}$ ) than the CaloJets, and the difference is particularly pronounced in the low jet $p_{\mathrm{T}}$ region.
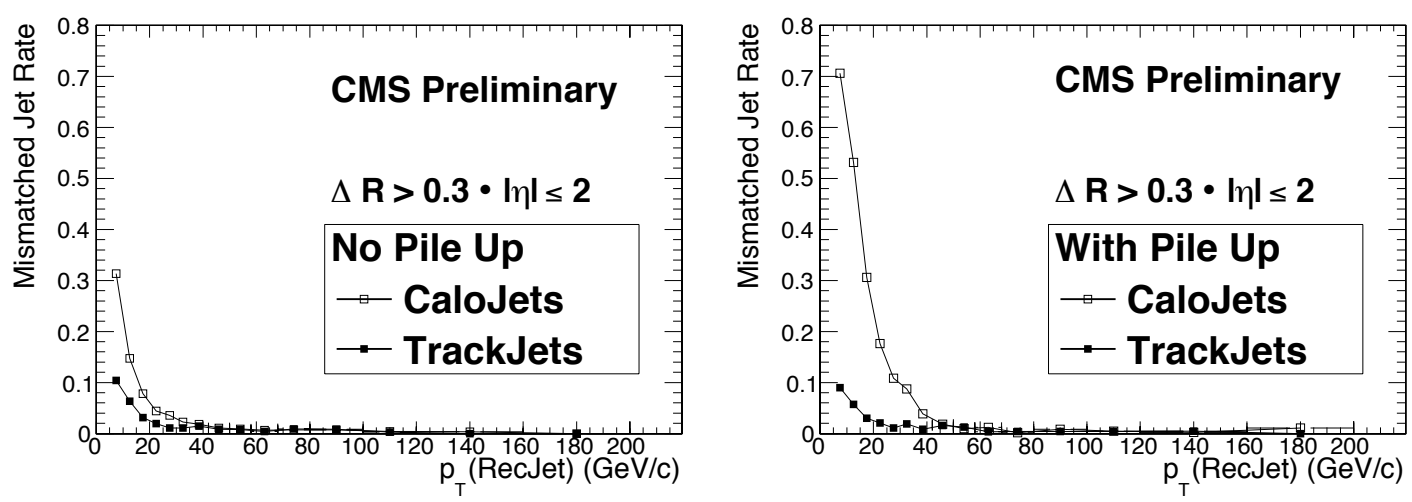

Figure 3: Jet mismatched rate in Z+jets events, as a function of the reconstructed jet $p_{\mathrm{T}}$, where signal events are without or with pile-up events.

To study the effect of pile-up, the signal samples of Z+jets are overlayed with a sample of Minimum Bias events where the mixing proportion is 1:5 per bunch crossing on average.

Tracks are measured at the interaction point and the information about the vertex of origin is therefore naturally taken into account. This information can be used to reject tracks that are not compatible with the primary interaction point, in this case the di-muon vertex.

Figure 3 also shows the jet mismatched rate after the pile-up events are included and reveals that the mismatched rate for TrackJets is not affected by the addition of pile-up events, while it increases significantly for CaloJets.

\section{Conclusions}

The performance of jet reconstruction with charged tracks only in CMS (TrackJets) has been presented and compared to calorimeter jets (CaloJets). Within the tracker acceptance, the jet finding efficiency with TrackJets is higher than for CaloJets for $p_{\mathrm{T}} \leq 30 \mathrm{GeV} / \mathrm{c}$, and the angular resolution of TrackJets is better than for CaloJets at $p_{\mathrm{T}} \leq 200 \mathrm{GeV} / \mathrm{c}$, mainly for the azimuthal $\phi$ resolution.

In multi-jet events, such as $t \bar{t}$ decays to six jets, the performance of jet finding with TrackJets is better than with CaloJets when considering the efficiency to match correctly the six quarks from the top decays, while setting the mismatched rate to the same level.

In $\mathrm{Z}+$ jets events TrackJets provide a simple and clean way for jet counting. Using the track vertex information the reconstruction of TrackJets is not affected by pile-up events.

\section{References}

[1] CMS Collaboration, Physics Technical Design Report, CERN-LHCC-2006-001

[2] CMS Collaboration, Performance of Jet Algorithms in CMS, PAS JME-07-003 (2007)

[3] CMS Collaboration, Performance of Jet Reconstruction with Charged Tracks only, PAS JME-08-001 (2008) 